\title{
INTEGRATED CHEMICAL SENSORS BASED ON CARBON BLACK AND POLYMER FILMS USING A STANDARD CMOS PROCESS AND POST-PROCESSING
}

\author{
Jeffrey A. Dickson and Rodney M. Goodman \\ Department of Electrical Engineering, \\ California Institute of Technology \\ Pasadena, California 91125 \\ dickson@caltech.edu
}

\begin{abstract}
We present an integrated chemical sensor array fabricated using a CMOS process followed by post-processing. The sensor presented in this paper incorporates 324 individually addressable sensing nodes. Post processing involves an electroless Nickel and Gold plating step to fabricate sensing contacts, and the deposition of a carbon black based polymer sensor material. The operation of the integrated sensor is confirmed. This sensor technology will allow the creation of large arrays of chemically diverse sensors.
\end{abstract}

\section{INTRODUCTION}

Arrays of chemical sensors have a number of advantages over discrete or small numbers of sensors. By enabling the creation of a large number of sensors we can approach the sensor count of the biological system. In addition, multiple sensors for the same odorant improves the redundancy of the system. By integrating chemicals sensors we can reduce its size, enabling the implementation of a large number of sensor sites. Moreover, a smaller sensor should be more sensitive, as a single odorant molecule will affect a larger percentage of the sensor area. Fabricating amplifiers in close proximity to the sensors enables addition of gain to the signal with minimal noise interference. Signal processing performed in parallel across the array can minimize extraneous inputs or detect features in the sensor responses, minimizing the data requirements.

This paper describes the development of an array of chemical sensors using carbon black sensors. The sensors are based on the conducting polymer approach of Lewis et al. [2-5], employing carbon black and non-conducting polymers. Carbon black particles mixed with the polymer, as opposed to polymerization, create a conducting polymer film. The number and density of the carbon black particles determines the electrical resistance of the polymer. When odor molecules absorb into the polymer the polymer swells, and

This work was supported by DARPA under DAAK6097K 9503.

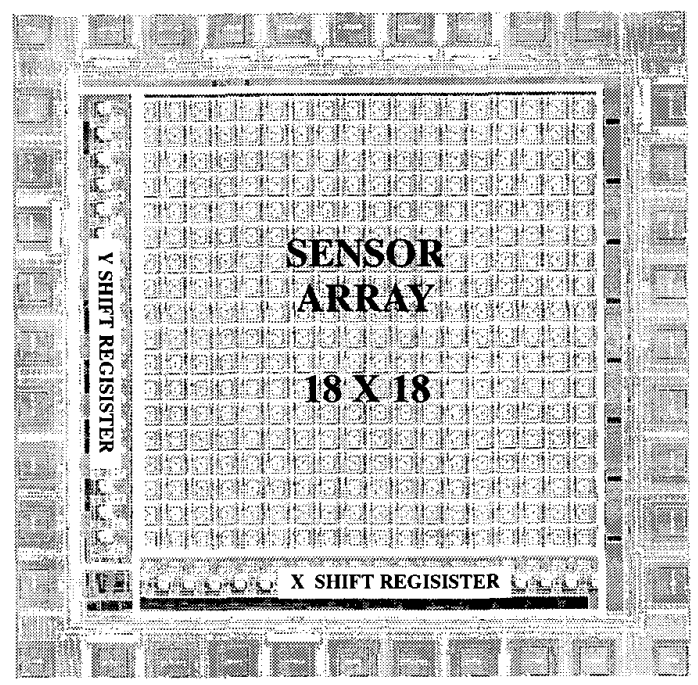

Figure 1: Block diagram of array chip. Fabricated in a 1.2 micron process, the chip contains 324 individual sites for chemical sensors.

the distance between the carbon black molecules increases. This in turn increases the electrical resistance of the polymer. Different polymers swell for different odor molecules, enabling the fabrication of a large number of different sensor types. These sensors make a very attractive candidate for integration with active circuitry, since they are easy to fabricate and present the detected signal in a form readily processed by circuitry.

The integrated sensor presented in this paper consists of an array of individually addressable sensor nodes. The chip, shown in Figure 1, consists of 324 sensors arranged in an $18 \times 18$ array. Previous array implementations have been limited to only a few sensors (for example [6]), and are not integrated with active circuitry. 


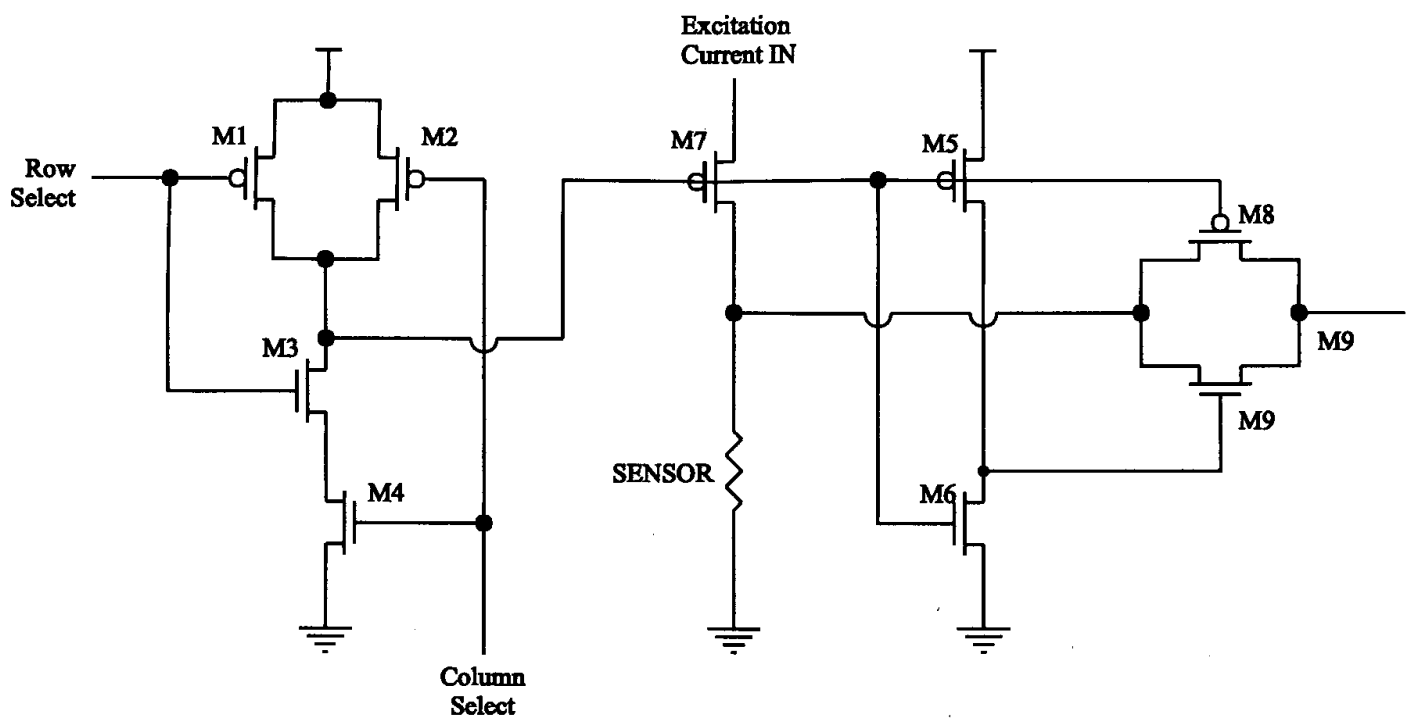

Figure 2: Schematic of the sensing unit cell. The sensor is shown as a resistor, excited by a current that is switched by $M_{7}$

\section{SENSOR PIXEL CIRCUITRY}

Figure 2 shows the schematic of the sensor unit cell. A NAND gate $\left(M_{1-4}\right)$ decodes the select lines generated at the edge of the array. This decoded signal drives a single transistor $M_{7}$ connected to the sensor, which switches a current generated at the bottom of the column. By switching the excitation current power consumption is minimized. To minimize noise the switch transistor should be as large as possible. With this scheme the selection lines only have to drive the smaller capacitance of the decoding logic. The output of the sensor is connected to a column output line by the transmission gate formed by $M_{8,9}$.

Since scanning through the array in a sequential manner is satisfactory for this design, the $\mathrm{X}$ and $\mathrm{Y}$ selection signals are generated by simple shift registers. The selection bit is loaded into the first stage of the register and is shifted along to select each consecutive row or column. The shift registers used for this chip were based on the scanner frame developed for silicon retinas [1].

\section{SENSOR DESIGN}

The deposition of the sensor material is complicated by nonuniformity of the carbon black distribution within the sensor material. After deposition of the solvent, polymer, and carbon black solution, the carbon black particles redistribute as the solvent evaporates. The carbon black particles are pushed to the edges of the drop, leaving a dense region of carbon black around the perimeter.

This "coffee ring" has a serious consequence on the phys- ical design of the sensor. The higher density of carbon black in the ring of the sensor will form a low resistance path between the contacts. Since this path is a very small portion of the resistor's area, it will not contribute greatly to the area available for sensing. However, the low resistance path will dominate the resistance, reducing the signal from the sensing area.

The sensor layout we used consisted of two terminals organized in a ring structure, 135 microns on a side. One sensor contact is located at the center of the ring, while the peripheral ring is the other contact. By making the exterior terminal common to all sensors, this conductor can be shared among all sensors. In addition, the aggregation of carbon black particles on the exterior of the sensor changes from a problem to a benefit. The higher carbon black density should help decrease the contact resistance between the sensor and the conductor. We are currently investigating modified topologies to mitigate the impact of layout on $1 / \mathrm{f}$ noise due to the non-uniform electric field [7].

\section{POST FABRICATION}

Our chips are processed in commercial foundries through the MOSIS service. Unfortunately, we are not able to simply take our chips and deposit the sensor material on the exposed aluminum. While aluminum is an adequate conductor of electricity, it also rapidly grows a native oxide on its surface in air. Post-fabrication processing of the chips is required to create suitable contacts for the sensor material.

Due to the prohibitive costs of obtaining full wafers, this contact creation has to be performed on individual die. Our 

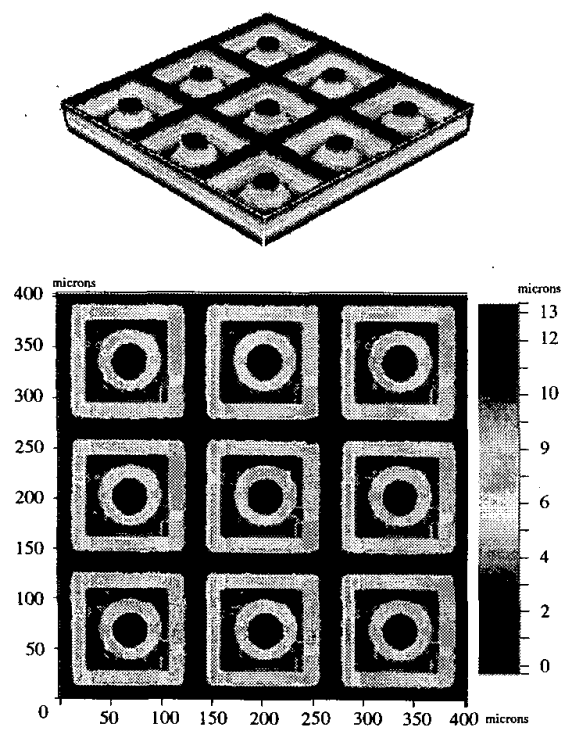

Figure 3: Profilometry of a $3 X 3$ section of array after the post-fabrication processing. The plating is approximated 10 microns high, and each sensor is 135 microns on a side. The internal contact is connected to the switch transistor, while the common perimeter contact is ground.

initial prototypes are only $2 \mathrm{~mm}$ on a side, making the evaporation and patterning of a conductor such as gold extremely difficult. For this reason we elected to use an electroless process that required no masking.

While electroless plating of aluminum is not a new technology, formulations compatible with integrated circuits are a recent development. The process we used is a proprietary formulation from Stapleton Technologies (Long Beach, California). The plating process required only seven procedures and was performed on a temperature controlled hotplate under a fume hood. The individual die were attached secured to glass slide using viton o-rings to facilitate handling.

After initial surface preparation steps, there are three main plating phases. The initial phase deposits Nickel, which is followed by a monolayer of gold and then a final gold plating step. We plated approximately 10 microns of Nickel, followed by 1 micron of gold. The Nickel plating forms wells on the surface of the chip to assist in containing the sensor material during deposition. The gold provides a nonreactive contact for the sensor. We plated to the 1 micron thickness to facilitate bonding to chip pads to the packaging after plating. Figure 3 shows a profilometry measurement of a $3 \times 3$ section of the array after plating, indicating approximately 10 microns of plating.

\section{SENSOR DEPOSITION}

With the sensor arrays successfully fabricated and plated, the next step is the deposition of the polymer material. For these initial tests a very simple deposition apparatus was employed: a paintbrush. The carbon black used in the sensor fabrication was a furnace black from Cabot Co. (Billerica, MA). To prepare the polymer-carbon black solution we added $20 \mathrm{mg}$ of carbon black and $80 \mathrm{mg}$ of the desired polymer, into $10 \mathrm{~mL}$ of the solvent. We sonicate the mixture for five minutes prior to deposition to suspend the carbon black particles.

Figure 4 shows a section of $3 \times 3$ sensors after deposition of the carbon black and polymer mixture. The "coffee ring" effect is clearly evident around the edges of the gold contacts. In this instance the higher density of carbon black improves the contact to the gold.

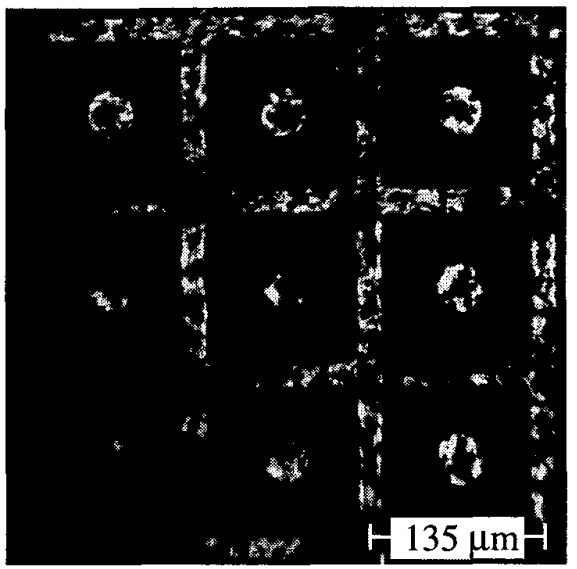

Figure 4: Sensor material deposited on a plated chip. The active circuitry is located underneath each sensor.

\section{TESTING}

The current vs, voltage curve for an individual sensor node is shown in Figure 5. The data show that the output is essentially linear. The deviation from linear is due to the nonlinearity of the on-chip output buffer, and not the sensor itself.

Figure 6 shows the output of a sensor made from the polymer poly(ethylene-co-vinyl acetate) reacting to exposure to Octanol. The resistance increases upon exposure to the octanol and reaches a new equilibrium. After removing the octanol the resistance returns to the original value. This confirms the successful integration of the sensor material and a standard CMOS process after post-fabrication processing. 


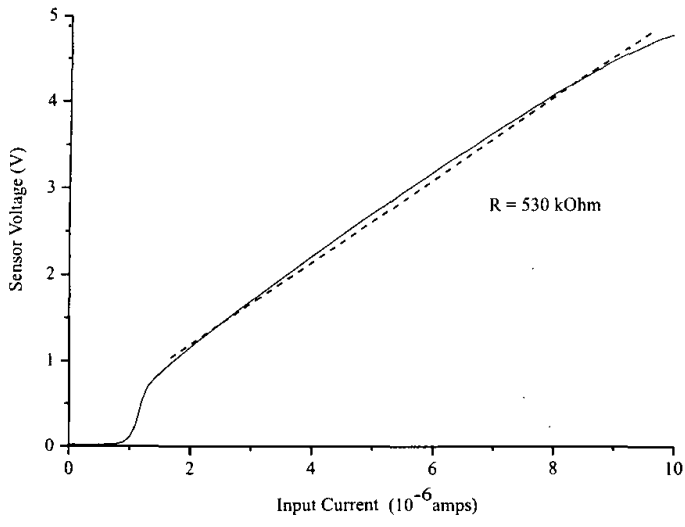

Figure 5: I vs V curve for an individual sensor. The nonlinearity is due to the column buffer amplifier.

Figure 7 shows the results for a single sensor exposed to a range of concentrations. We exposed the chip to vapors of acetone, methanol, THF, and toluene in concentrations of $2 \%, 4 \%, 6 \%$ and $8 \%$ and measured the response of a sensor fabricated from poly-sulfane dissolved in THF. The sensor response, given by the percentage change in resistance, has a linear relationship to concentration.

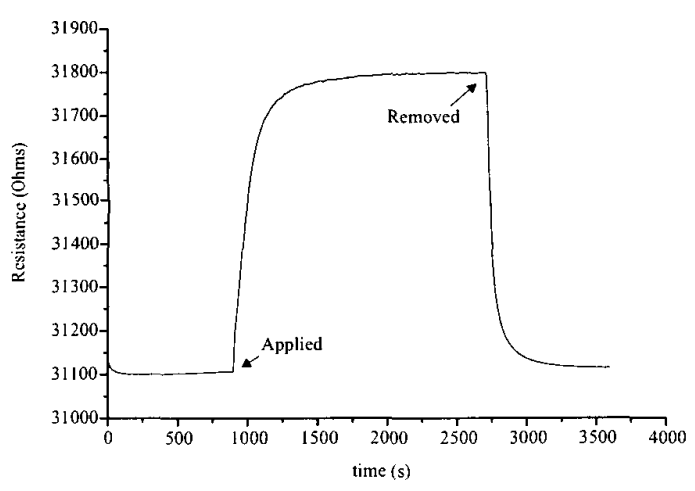

Figure 6: Carbon Black sensor detecting Octanol. The resistance increases during the exposure.

\section{CONCLUSION}

We have demonstrated the successful integration of a standard CMOS process with a carbon black based polymer sensor. We are currently combining this technology with the large number of chemically selective polymer sensors available [3] to create large arrays of chemically diverse sensors capable of chemical classification. We are also investigating more advanced circuitry for each sensor site, such as gain, adaptation of baseline drift, and ratiometric sensing.

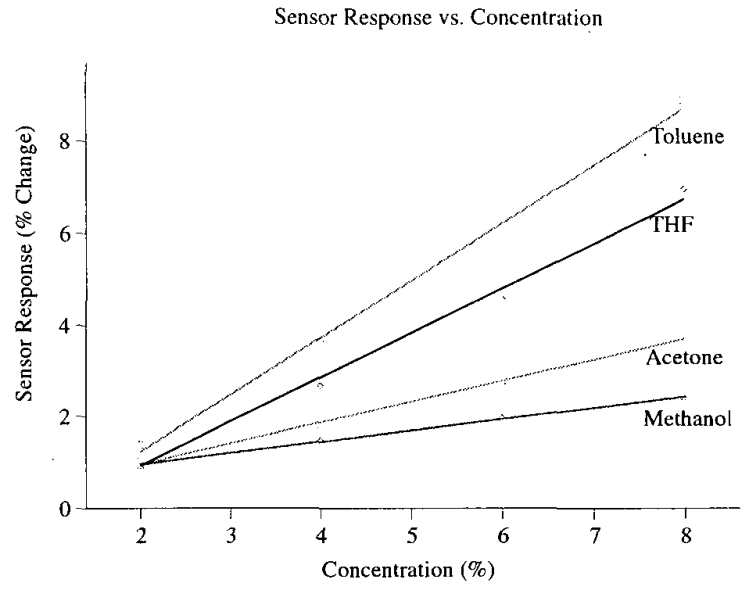

Figure 7: Concentration response of a sensor. The response of the sensor is linearly proportional to the concentration.

\section{ACKNOWLEDGEMENTS}

The authors would like to thank Charles Grosjean for his assistance with the profilometery measurements, Tom Tsao and Phil Stapleton for their input on the plating procedure, and Nate Lewis, Erik Severin and Shawn Briglin for their assistance with the chemical sensors.

\section{REFERENCES}

[1] T Delbruck and C.A. Mead. Scanners for visualizing activity of analog VLSI circuitry. Analog Integrated Circuits and Signal Processing, 1:93-106, 1991.

[2] B. J. Doleman, M. C. Lonergan, E. J. Severin, T. P. Vaid, and N. S. Lewis. Quantitative study of the resolving power of arrays of carbon black-polymer composites in various vaporsensing tasks. Anal. Chem., 70(19):4177-4190, 1998.

[3] B. J. Doleman, R. D. Sanner, E. J. Severin, R. H. Grubbs, and N. S. Lewis. Use of compatible polymer blends to fabricate arrays of carbon black-polymer composite vapor detectors. Anal. Chem., 70(13):2560-2564, 1998.

[4] B. J. Doleman, E. J. Severin, and N. S. Lewis. Trends in odor intensity for human sind electronic noses: Relative roles of odorant vapor pressure vs. molecularly specific odorant binding. Proc. Natl. Acad. Sci. U. S. A., 95(10):5442-5447, 1998.

[5] M. S. Freund and N. S. Lewis. A chemically diverse conducting polymer-based electronic nose. Proc. Natl. Acad. Sci. U. S. A., 92(7):2652-2656, 1995.

[6] J. W. Gardner, A. Pike, N. F. Derooij, M. Koudelkahep, P. A. Clerc, A. Hierlemann, and W. Gopel. Integrated array sensor for detecting organic-solvents. Sens. Actuator B-Chem., 26(13):135-139, 1995.

[7] H. Yoshida. The Effect of Resistor Geometry On Current Noise. IEEE Transactions On Components Hybrids and Manufacturing Technology, 16(3):344-349, 1993. 